\title{
Prolonged Exposure Dermatosis: Reporting High Incidence of an Undiagnosed Facial Dermatosis on a Winter Wilderness Expedition
}

\author{
Jodie E. Totten, MD; Douglas M. Brock, PhD; Tod D. Schimelpfenig; Justin L. Hopkin, MD; Roy M. Colven, MD \\ From the Division of Emergency Medicine (Dr Totten) and Department of Family Medicine (Dr Brock), University of Washington, Seattle, WA; the \\ Wilderness Medicine Institute of the National Outdoor Leadership School, Lander, WY (Mr Schimelpfenig); the Department of Internal Medicine \\ (Dr Hopkin) and the Division of Dermatology (Dr Colven), University of Washington, Seattle, WA.
}

Objective.-Previously unclassified inflammatory skin lesions referred to as sun bumps have been observed throughout the year on participants of wilderness trips; however, the underlying cause and diagnosis remain unclear. The purpose of this prospective observational study was to document the incidence, characteristics, and risk factors associated with these skin lesions as they occurred on a winter wilderness expedition.

Methods.-For this study, the lesions were defined as pruritic or erythematous skin lesions occurring while in the wilderness. Seventy-four participants in a wilderness ski touring course in Wyoming fully completed a 44-question written survey concerning occurrence and risk factors for these lesions. Weather information and photographs were collected.

Results.-Twenty-six percent of participants had similar lesions. The lesions were described as edematous pale papules and plaques with erosions and crusts on an erythematous background. The face was involved in $90 \%$ of affected persons. Lesions occurred after an average of 8.7 days in the wilderness and resolved 10.6 days later. Skin that was less prone to sunburn was associated with a decreased incidence (odds ratio 0.44). No association could be found between lesion incidence and history of polymorphous light eruption, sun exposure, ambient temperature, affected contacts, sex, or body mass index.

Conclusions.-Overall, the lesions were common among study participants but occurred only after prolonged exposure to wilderness conditions. It was not possible to classify the skin condition as an example of any known diagnosis. We propose the name "prolonged exposure dermatosis" for this condition until further studies better define its etiology, prevention, and treatment.

Key words: wilderness, dermatology, cold exposure, sun exposure

\section{Introduction}

Dermatologic complaints in the wilderness are both common and relatively understudied, making the diagnosis of such conditions difficult. ${ }^{1,2}$ The National Outdoor Leadership School (NOLS) is a large, international, wilderness education program that has taken more than 120,000 students into the wilderness since its founding in $1965 .^{3}$ For many years, NOLS staff members have noted the occurrence of lesions they refer to as "sun bumps." They have noted these lesions to occur throughout the year, both during and outside of NOLS

Corresponding author: Jodie E. Totten, MD, Harborview Medical Center, 325 9th Avenue, Box 359702, Seattle, WA 98104-2499 (e-mail: jet25@uw.edu). trips, and to affect mainly the hands and the face. Some affected persons believed the lesions were caused by sun exposure, which led to the colloquial term. At the request of NOLS staff, this study was designed to document these lesions to assist in their diagnosis, treatment, and prevention. Before this study, these lesions were thought to most likely be a variant of polymorphous light eruption (PMLE) because PMLE is the most common intrinsic photodermatosis; however, the diagnosis has remained unclear. ${ }^{4}$

\section{Methods}

Study lesions were defined as pruritic or erythematous skin lesions occurring while in the wilderness. Although 
the condition has been noted during summer and winter courses, for this study, observations during a single winter wilderness expedition were recorded. All 89 students and instructors on the 2011 Spring Semester in the Rockies course run by NOLS were approached to be part of the study before leaving for the first wilderness section. Participation in the study did not affect student grading or participation in the course. Informed written consent was obtained from 62 students (89\%) and from all 19 instructors (Figure 1). Study participants were assigned unique study codes that were known only to NOLS staff and the researchers.

The course was operated as 6 separate groups consisting of 10 to 12 students and 3 instructors. All groups completed a 10-day ski touring expedition through the mountain ranges of Wyoming. All groups traveled at similar elevations for the same number of expedition days and snow camping. Specific routes varied from group to group. While on the course, students shared tents, food preparation, and dinner with 2 to 3 other students within their group, known as their "tent group." Instructors recorded the daily temperature and cloud cover. They also photographed skin lesions during the course.

On returning to Lander, Wyoming, $92 \%$ of students $(n=57)$ and $89 \%$ of instructors who had given consent $(n=17)$ fully completed a 44-question, 10 - to 15 -minute survey concerning demographics, skin type, sunscreen use, protective wear, medical history, medication use, experience with skin lesions before and during the trip, and any skin lesion treatments. Participants were asked to categorize their skin type by propensity to sunburn vs tan into 1 of 4 gradated categories. This survey was previously piloted with 5 NOLS staff members familiar with this skin condition. Participants who had skin lesions during the course had their skin examined and photographed by a medical student (J.T.) and a physician (J.H.). Sunscreens and creams used on skin affected by lesions were recorded. When the date of lesion onset was described as a range (eg, "day 5 to day 6"), the mean (eg, day 5.5) was used for data analysis.

The results were analyzed in IBM SPSS Statistics 19 using Pearson's $\chi^{2}$ analysis, analysis of variance, and descriptive statistical tests. A logistic regression was performed using sex, skin type, body mass index (BMI), and the interaction variable of sex and BMI. Photographs and interview information were reviewed and discussed with 2 University of Washington faculty dermatologists (R.C., and Dr Kyle Garton) who were not otherwise associated with NOLS and who had no previous personal or clinical experience with these lesions.

This study was approved by the University of Washington Institutional Review Board. A literature review to identify the documented lesions included, but was not limited to, PubMed.gov searches for articles in English and available through the University of Washington using the following search terms: wilderness dermatology, wilderness dermatosis, photodermatosis, exposure dermatosis, expedition dermatoses, sun bumps, nonfreezing cold injury, cold injury dermatoses, cold injury dermatosis, cold facial lesions, wind dermatosis, wind dermatoses, facial frostbite, expedition dermatosis, chilblains, pernio, cold panniculitis, mountaineering rash, mountaineering dermatosis, mountaineering skin lesions, plaque ulceration face, hypopigmented plaque face, and edematous facial plaque. Review articles and textbooks describing polymorphic light eruption, pernio (chilblains), cold panniculitis, cold urticaria, and solar urticaria were reviewed. Wilderness Medicine, Sixth Edition, by Paul S. Auerbach, and numerous dermatologic text books were also searched.

\section{Results}

\section{COURSE CLIMATE CHARACTERISTICS}

The average high temperature for all sites on all days was $-5^{\circ} \mathrm{C}$. The average low temperature was $-10^{\circ} \mathrm{C}$. Temperatures reported ranged from $-28^{\circ} \mathrm{C}$ to $10^{\circ} \mathrm{C}$. Fifty-one percent of days were sunny or partially sunny, and $49 \%$ were cloudy or snowing. The altitude for the courses varied between $2400 \mathrm{~m}$ and $3350 \mathrm{~m}$.

\section{DEMOGRAPHICS}

The demographics of participants are provided in Table 1. Of the 70 students and 19 instructors participating in the NOLS course, 57 students (81\%) and 17 instructors (89\%) completed all aspects of the study, for an overall participation rate of $83 \%$. Twenty-three percent of participants were female. Student ages ranged from 18 to 30 years, and instructor ages ranged from 24 to 56 years. Prior skin conditions included rash after sun exposure without history of PMLE $(n=7)$, PMLE $(n=5)$, atopic dermatosis $(n=5)$, and rosacea $(n=1)$. No participants had a history of lupus, porphyria, seborrheic dermatosis, or other photo-exacerbated skin disease.

\section{INCIDENCE AND RISK FACTORS}

Of all participants, 19 (26\%) reported lesions (Table 1). The incidence was similar between men (13 [23\%]) and women (6 [35\%], $P=.30$ ) as well as between students (16 [28\%]) and instructors (3 [18\%], $P=.39$ ). No affected person had a history of PMLE. Frequency of sunscreen use and the use of a brimmed hat was not associated with a change in incidence. Overall, $77 \%$ of 


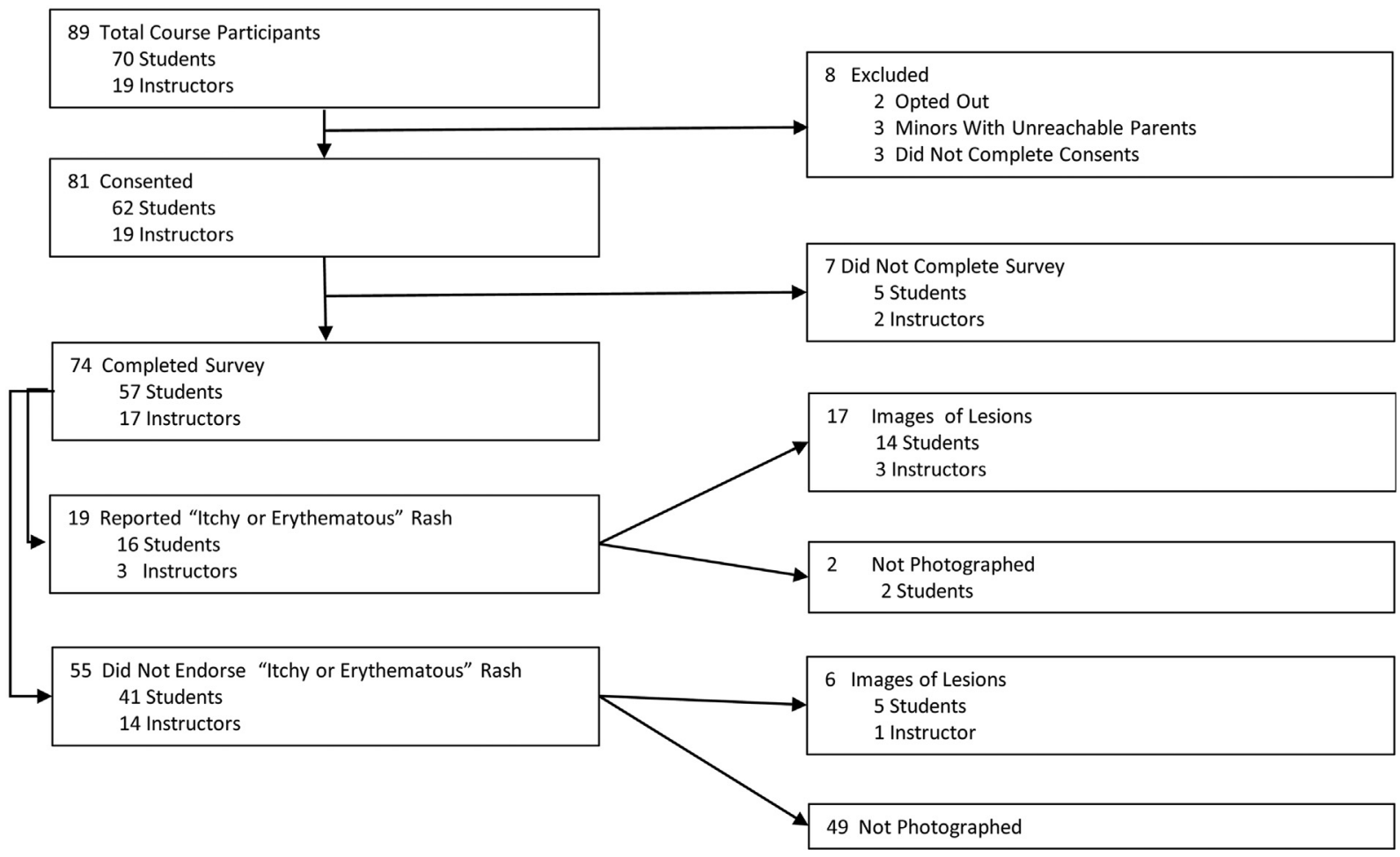

Figure 1. Consort diagram.

Table 1. Risk factors

\begin{tabular}{|c|c|c|c|}
\hline Risk factors & $P E D$ & No PED & Total \\
\hline Total participants & $19(26)$ & $55(74)$ & 74 \\
\hline Instructors & $3(18)$ & $14(82)$ & 17 \\
\hline Students & $16(28)$ & $41(72)$ & 57 \\
\hline Female & $6(35)$ & $11(65)$ & 17 \\
\hline Male & $13(23)$ & $44(77)$ & 57 \\
\hline \multicolumn{4}{|l|}{ Skin type } \\
\hline Always sunburns, never tans & $3(43)$ & $4(57)$ & 7 \\
\hline Usually sunburns, sometimes tans & $10(32)$ & $21(68)$ & 31 \\
\hline Seldom sunburns, usually tans & $6(18)$ & $28(82)$ & 34 \\
\hline Never sunburns, always tans & $0(0.0)$ & $2(100)$ & 2 \\
\hline History of diagnosed PMLE & $0(0.0)$ & $5(9.3)$ & 5 \\
\hline History of sun-related rash without diagnosed PMLE & $7(37)$ & $10(18)$ & 17 \\
\hline Any other skin condition & $3(16)$ & $7(13)$ & 10 \\
\hline \multicolumn{4}{|l|}{ Frequency of sunscreen use } \\
\hline Less than daily & $7(23)$ & $24(77)$ & 31 \\
\hline Daily & $7(30)$ & $16(70)$ & 23 \\
\hline Multiple times a day & $5(28)$ & $13(72)$ & 18 \\
\hline \multicolumn{4}{|l|}{ Brimmed hat use } \\
\hline Never & $5(19)$ & $22(82)$ & 27 \\
\hline Sometimes & $6(32)$ & $13(68)$ & 19 \\
\hline Usually & $5(31)$ & $11(69)$ & 16 \\
\hline Always & $3(27)$ & $8(73)$ & 11 \\
\hline
\end{tabular}

Values are $n(\%)$.

PED, prolonged exposure dermatosis; PMLE, polymorphous light eruption. 
Table 2. Lesion characteristics

\begin{tabular}{lc}
\hline Time course & \\
Onset, days & $8.7(4.5-11)$ \\
Duration, days & $10.6(5-16)$ \\
Locations affected & \\
Area covered by clothing & $2(11)$ \\
Symmetric areas of body & $15(80)$ \\
Face & $17(90)$ \\
Ears & $4(21)$ \\
Hands & $0(0)$ \\
Lesion characteristics & \\
Pruritic & $13(68)$ \\
Blistering & $8(44)$ \\
Swelling & $13(68)$ \\
Painful & $3(16)$ \\
Treatment used & \\
Increased clothing coverage & $9(47)$ \\
Cream or ointment & $11(58)$ \\
Covered lesions with tape & $2(11)$ \\
Other (zinc oxide) & $1(7)$ \\
\hline
\end{tabular}

Values are mean (range) or $n(\%)$.

participants reported using sunscreen with at least 30 sun protection factor. One affected person had applied zinc oxide daily. There was no significant exposure difference in the number of sunny vs cloudy days or average temperatures between affected and unaffected participants. There were also no significant variations in incidence between tent and traveling groups to suggest infectivity. Of 4 participants listing nonsteroidal antiinflammatory drug use, none had the lesions. One of the 2 participants taking erythromycin was affected. The only participant taking minocycline was also affected. No participants reported doxycycline or sulfonamides use.

\section{LOGISTIC REGRESSION ANALYSIS}

Logistic regression with an interaction term (BMI by sex) was used to analyze the association between lesion incidence and sex, skin type, and BMI. Skin less prone to sunburn was associated with a decreased incidence of the lesions (odds ratio [OR] 0.44, 95\% CI: 0.19-1.0, $P=.05)$. There was no significant association between lesion incidence and sex (OR 2.2, 95\% CI: $0.12-39$, $P=.60)$ or BMI (OR $1.5,95 \%$ CI: $0.23-11, P=.66$ ). The BMI by sex interaction was not significant.

\section{CHARACTERISTICS OF LESIONS}

On examination, lesions were edematous hypopigmented papules and plaques on an erythematous background (Table 2; Figures 2 and 3). They frequently exhibited central erosions and crusts, or vesicular or pseudovesicular change. The lesions were most frequently seen on the cheeks and nose (90\%); less often, the ears were involved $(21 \%)$. Most of the recorded lesions were consistent in morphology from one participant to the next. However, 1 man had lesions on his ears and a history more consistent with healing frostbite. Because other survey data were not modified based on interview data, this subject was not removed from the survey data analysis. Based on the photographs, surveys, examination information, and clinical information, the lesions could not be assigned a diagnosis by the clinical dermatologists.

Although the term sun bumps has been used within NOLS informally to refer to lesions that could occur either on the face or on the hands, no hand lesions were observed during this study. However, it should be noted that all respondents in this study reported wearing gloves "usually" or "always" during this course. Other reported systemic symptoms (muscle aches, fever and chills, joint aches or pains, nausea, vomiting, or diarrhea) did not vary between affected and unaffected participants.

Several participants tried keeping the affected areas covered with clothing, increasing sunscreen use, or using zinc oxide to prevent further lesions or to treat existing lesions. Two applied tape to prevent further exposure of the area. One participant applied hydrocortisone cream. The only participant who felt intervention had improved the lesions had increased clothing coverage to the area. No oral medications were tried for treating the skin condition.

\section{PREVIOUS LESION EXPERIENCE}

Of participants who had lesions during this course, 5 (26\%) had previous experience with similar lesions. Of these 5 participants, $4(80 \%)$ had lesions affecting the same area on this course as had been affected on prior occasions. No one reported lesion scarring or worsening of lesions with reoccurrence. Lesions had previously occurred in snow/glacial environments for 3 participants (75\%), desert environments for $2(40 \%)$, and river/ocean environments for $1(20 \%)$. No respondent had sought medical attention from a healthcare provider for these lesions.

\section{Discussion}

This study provides a systematic description of and search for associated factors of a cutaneous condition commonly recognized within NOLS expedition participants. In the opinion of 2 academic dermatologists, this condition could not be diagnosed as any known skin disease based on history and photographs. Although 


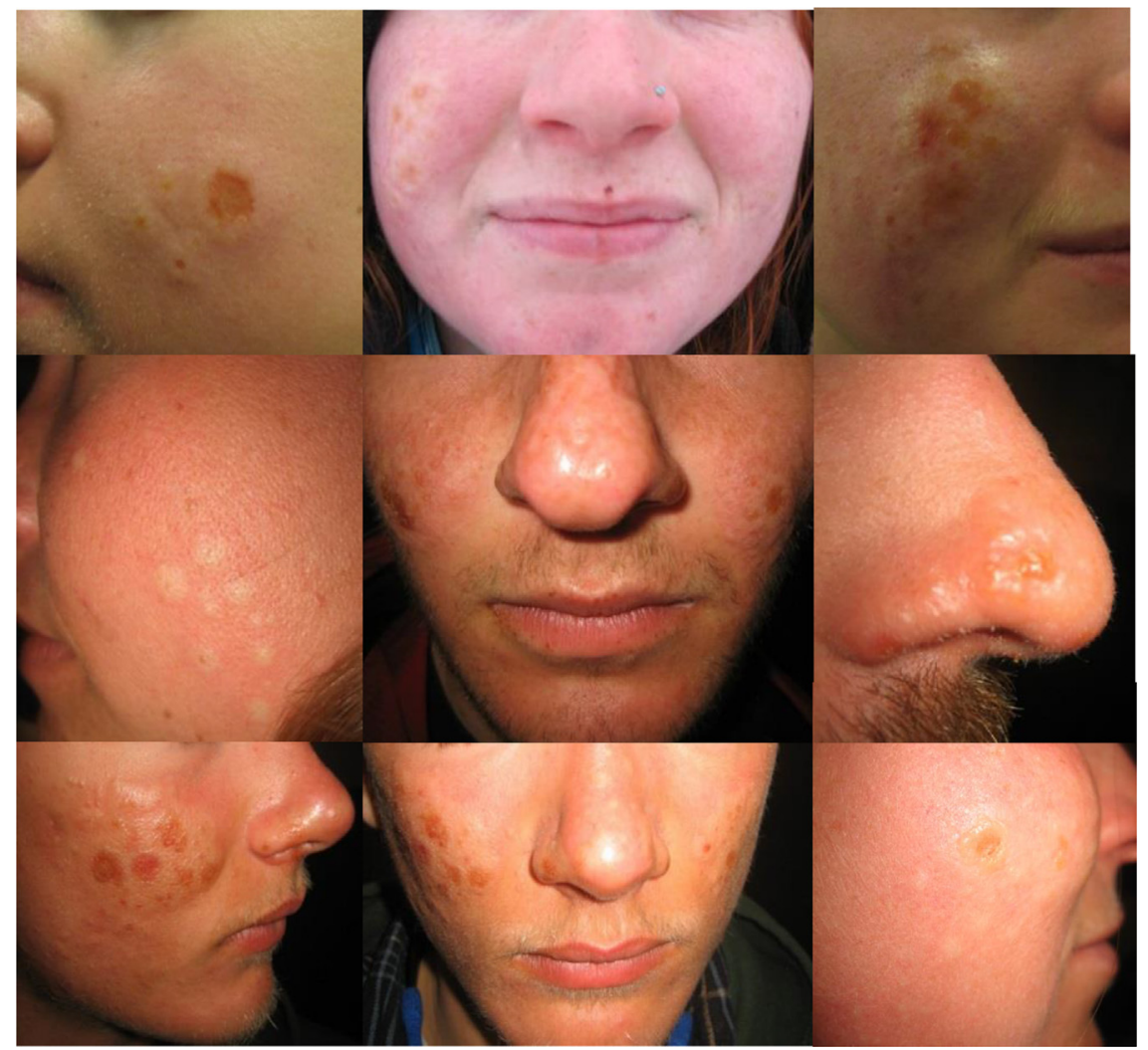

Figure 2. Examples of lesions that occurred during the study period.

ultimately no etiology of the lesions could be named, we are now better able to compare the characteristics of these lesions with other known skin conditions. Before this study, photo-induced or exacerbated conditions, particularly PMLE and drug-induced photosensitivity, were thought likely to be responsible for this condition because of frequent exposure to sunlight during most expeditions. However, none of the participants with a previous diagnosis of PMLE developed lesions on this course. The delayed onset at 8.7 days would also be unusual for PMLE. ${ }^{4}$ There was no consistent correlation with photosensitizing drugs. Moreover, these lesions did

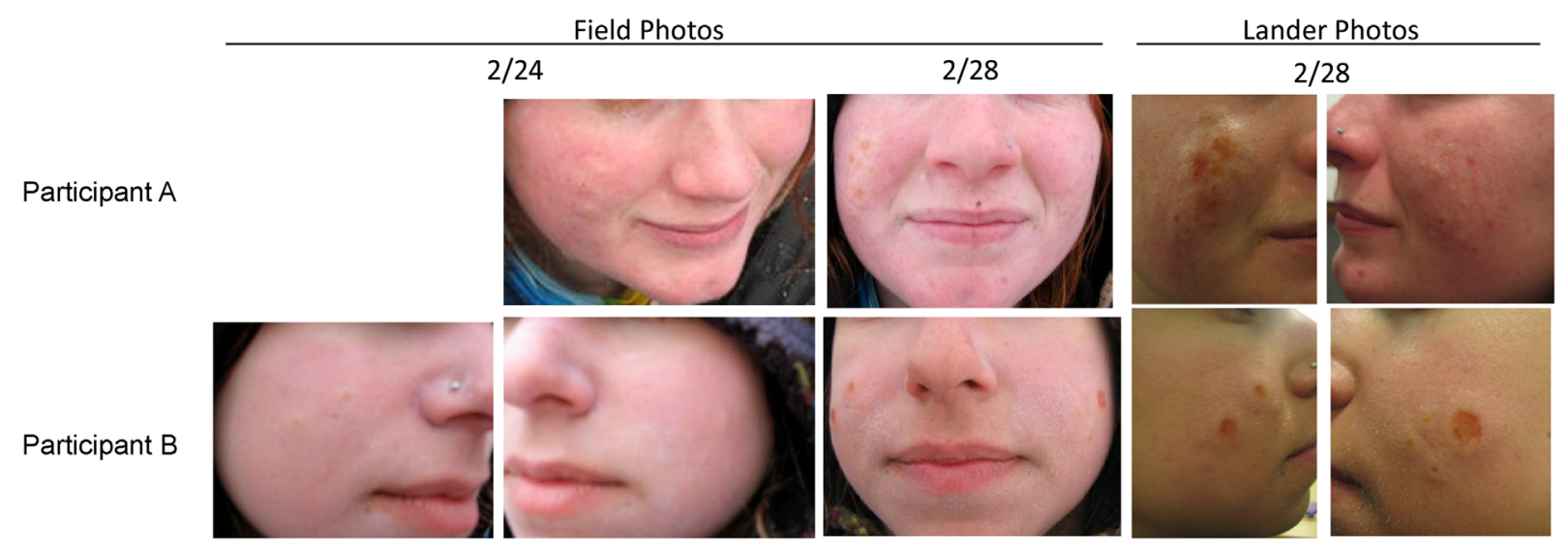

Figure 3. Progression of the lesions showing both field photographs and photographs after return to Lander. 
not correlate with the number of sunny days and were not associated with decreased use of sunscreen or protective clothing; 1 affected participant had the lesions despite daily application of sunscreen and zinc oxide to and clothing coverage of the affected area. Together, that argues against the role of sunlight as an associated factor in inducing these lesions.

When compared with common cold-related injuries, the lesions in this study were nontender, pale, and surrounded by a blanchable erythema, which does not align with the violaceous, purpuric, and usually tender lesions of pernio (chilblains). ${ }^{5}$ Additional exposureassociated diagnoses include solar urticaria or cold-induced urticaria. However, that would be an unusual presentation for urticarial lesions, as the lesions were delayed in onset and persistent over time.

Cold panniculitis might also be considered and has been reported to affect the cheeks. In this condition, lesions appear quickly on exposure of the skin to cold and have a violaceous color, ${ }^{6}$ in contrast to the lesions in the present study that were delayed in onset and presented with blanchable erythema. The season and distribution of the lesions are not consistent with an arthropod bite reaction. Tumid lupus erythematous can cause similar lesions, although such a high random prevalence of this rare autoimmune disorder on 1 trip would be unusual.

Although the lesions on this course do not clearly align with any of the above diagnoses, more definitive diagnosis or elimination of diagnoses would be aided by skin biopsies and histopathology, a logical area for further study of this condition. Furthermore, although hand lesions have been described by NOLS staff, none occurred during this course. Further studies of different expeditions, especially those taking place in different seasons and environments, may help to clarify this discrepancy.

\section{STUDY LIMITATIONS}

The ability of this study to elucidate risk factors was limited by the small study size. Histologic diagnosis of the lesions could not be made as biopsies were not collected. Underreporting of the lesions on the survey was noted, as a number of participants reported not having the defined lesions on the survey or did not answer that question but later arrived to have their lesions documented (Figure 1).

\section{Conclusions}

To our knowledge, this is the first study of prolonged exposure dermatosis. The lesions were found to be both common in this setting and poorly aligned with any known dermatological entities. No correlation could be found between sun exposure or lack of sun protection and lesion incidence. Lesion characteristics were also not consistent with known cold-induced conditions. Despite the absence of an etiological diagnosis, this study has helped to clarify possible risk factors for prolonged exposure dermatosis and to create a more objective description of this previously unreported condition.

\section{Acknowledgments}

The authors would like to thank the National Outdoor Leadership School in Lander, Wyoming, for donating their time and the use of their facilities as well as sharing their experience and knowledge; Kyle Garton, MD, of the University of Washington Division of Dermatology for providing additional dermatological expertise; the many NOLS instructors and staff who gave their time to help complete this study; and Nancy and Paul Ebbert for their support.

\section{References}

1. Price R, Murdoch D. Perniosis (chilblains) of the thigh: report of five cases, including four following river crossings. High Alt Med Biol. 2001;2:535-538.

2. McLaughlin KA, Townes D, Wedmore I, Billingsley RT, Listrom CD, Iverson LD. Pattern of injury and illness during expedition-length adventure races. Wilderness Environ Med. 2006;17:158-161.

3. National Outdoor Leadership School. History. Available at: http://www.nols.edu/about/history/nols_history.shtml. Accessed November 30, 2014.

4. Bylaite M, Grigaitiene J, Lapinskaite GS. Photodermatoses: classification, evaluation and management. $\mathrm{Br} J$ Dermatol. 2009;161:61.

5. Prakash S, Weisman MH. Idiopathic chilblains. Am J Med. 2009; $122: 1152$.

6. Quesada-Cortes A, Campos-Munoz L, Diaz-Diaz RM, Casado-Jimenez M. Cold panniculitis. Dermatol Clin. 2008;26:485. 